Relations industrielles

Industrial Relations

\title{
Possono migliorare le relzioni industriali in Italia? Par Gino Giugni, Luigi Mengoni, Felice Mortillaro et Dimitri Weiss, Torino, Gruppo Dirigenti Fiat, 1981, 140 pp.
}

\section{P. Joubert}

Volume 38, numéro 1, 1983

URI : https://id.erudit.org/iderudit/029344ar

DOI : https://doi.org/10.7202/029344ar

Aller au sommaire du numéro

Éditeur(s)

Département des relations industrielles de l'Université Laval

ISSN

0034-379X (imprimé)

1703-8138 (numérique)

Découvrir la revue

Citer ce compte rendu

Joubert, P. (1983). Compte rendu de [Possono migliorare le relzioni industriali in Italia? Par Gino Giugni, Luigi Mengoni, Felice Mortillaro et Dimitri Weiss,

Torino, Gruppo Dirigenti Fiat, 1981, 140 pp.] Relations industrielles / Industrial Relations, 38(1), 190-192. https://doi.org/10.7202/029344ar

Tous droits réservés @ Département des relations industrielles de l'Université Laval, 1983
Ce document est protégé par la loi sur le droit d'auteur. L’utilisation des services d'Érudit (y compris la reproduction) est assujettie à sa politique d'utilisation que vous pouvez consulter en ligne.

https://apropos.erudit.org/fr/usagers/politique-dutilisation/ 
Conférence internationale du travail. Cette nouvelle compilation ne constitue pas rigoureusement une seconde édition du recueil publié en 1966 puisque les conventions et recommandations ne sont plus présentées par ordre chronologique, mais bien par sujets.

L'ouvrage comporte deux tables des matières. La première suit l'ordre de représentation des instruments par sujets; la seconde, l'ordre chronologique. Ains, l'ouvrage pourra être aisément consulté tant par ceux qui cherchent à obtenir une information sur les normes de l'O.I.T. au regard d'un sujet donné, que par ceux qui, connaissant le numéro d'un instrument ou l'année de son adoption, désirent en consulter le texte.

Ce volume contient le texte de 146 conventions et 102 recommandations adoptées par la Conférence entre 1919 et 1981 . Les conventions qui ne figurent pas dans le recueil - dix au total - relèvent de trois catégories: les conventions qui n'ont pas reçu le nombre de ratifications nécessaire pour leur entrée en vigueur et qui ne sont plus ouvertes à la ratification à la suite de l'entrée en vigueur de conventions portant leur révision; les conventions qui n'ont pas encore reçu le nombre de ratifications nécessaire pour leur entrée en vigueur et qui furent révisées par une convention postérieure; les conventions portant révision des articles finals. Les soixante-trois recommandations non reproduites dans cette compilation ont été omises soit parce qu'elles ont été remplacées par une convention ou une recommandation postérieure, soit parce qu'elles sont de nature purement transitoire. Les différentes subdivisions de l'ouvrage comportent le cas échéant la liste des instruments non reproduits avec des renvois adéquats aux sources où les textes peuvent être consultés, soit au Compte rendu de la Conférence, soit au Bulletin officiel du B.I.T.

Les instruments intéressant plus d'un sujet sont reproduits dans la section la plus directement pertinente; la table des matières par sujets comporte à cet égard des renvois appropriés aux instruments publiés ailleurs dans le volume.
Enfin, signalons que seules les dispositions substantives des instruments sont reproduites. Les dispositions finales ainsi que les dispositions relatives aux déclarations d'application à des territoires non métropolitains sont reproduites dans deux appendices à la fin du volume. Sous chacune des conventions, des notes infrapaginales appropriées renvoient le cas échéant à ces appendices.

Compte tenu que la dernière compilation des instruments élaborés au sein de l'O.I.T. remontait déjà à 1966 , il n'est pas douteux que la publication de ce nouveau recueil sera saluée avec satisfaction par tous ceux qu'intéresse le développement du droit international du travail.

La version française de ce recueil sera publiée incessamment par le B.I.T.

Alain BARRÉ

Université Laval

Possono migliorare le relazioni industriali in Italia? par Gino Giugni, Luigi Mengoni, Felice Mortillaro et Dimitri Weiss, Torino, Gruppo Dirigenti Fiat, $1981,140 \mathrm{pp}$.

Le présent volume, dont le copyright est daté novembre 1981 mais qui a été, en réalité, publié en 1982, rassemble les actes de deux colloques successifs qui virent "face à face", respectivement en mai et en juin 1981, Felice Mortillaro, professeur chargé de cours à l'Université de Pérouse et directeur général de la Fédération patronale italienne de la métallurgie, Federmeccanica, et Gino Giugni, professeur titulaire de droit du travail à l'Université de Rome, co-auteur de la loi dite «Statut des travailleurs", pour le premier d'entre eux, et Dimitri Weiss, professeur titulaire de Sciences de gestion et relations industrielles à l'Université du Maine et à l'Institut d'Administration des Entreprises de l'Université de Paris I Panthéon-Sorbonne, et Luigi Mengoni, professeur titulaire de Droit civil à l'Université catholique de Milan, également co-auteur du Statut des travailleurs. 
F. Mortillaro, dans un dense exposé sur «la représentation des intérêts des travailleurs en Italie», point central des relations du travail dans ce pays, fait d'abord un survol historique de la question: le lien entre syndicats et cette représentation. Le système de représentation entre en crise, dit Mortillaro, non pas en 1968-1969 comme on le croit communément, mais au moment où la négociation d'entreprise devient un élément central de l'édifice contractuel. D'où tout le problème de la négociation articulée, de la création, la coexistence et, ultérieurement, la disparition des commissions internes et des sections syndicales d'entreprise, la naissance des conseils d'usine, mouvement ayant des objectifs différents de celui mis en place par le statut des travailleurs, qui prévoyait des représentations syndicales d'entreprise. Et au leader patronal de relier cette évolution à l'exercice du droit de grève et de la capacité nécessaire au déclenchement du conflit ouvert.

En résumé, la représentation des intérêts des travailleurs, loin d'être institutionnalisée, finit par se superposer sur plusieurs plans. Cette superposition a des conséquences sur l'initiative syndicale et sur l'utilisation par l'entreprise des outils de la négociation collective, le problème du lien entre grève et représentation des intérêts des travailleurs étant loin d'avoir été résolu.

G. Giugni traite de «systèmes de négociation", c'est-à-dire d'un système basé sur l'existence d'un certain nombre de règles du jeu, plus ou moins formalisées, ce qui suscite d'emblée la question de savoir si l'Italie vit ou non dans une condition de pure anomie, c'est-à-dire d'ingouvernabilité des relations du travail. Probablement ni système entier, ni anomie complète... Pays à bas degré de formalisation légale, l'Italie a en ce moment comme problème central celui relatif aux niveaux et domaines de la négociation. L' «automne chaud" avait eu comme conséquence l'affirmation d'un système contractuel à plusieurs niveaux, non coordonnés entre eux -et qui, de ce fait, n'est peut-être pas un vrai système-, qui se chevauchent les uns les autres, encore que le phénomène semble quelque peu décroissant.
Si le premier colloque a porté sur la représentation, le second eut comme thème le conflit. Il appartenait à Dimitri Weiss de poser le problème dans une perspective de relations industrielles internationales et comparées. Et d'emblée il s'interroge sur la définition du conflit industriel, sur la confusion opérée le plus souvent entre conflit du travail et grève, sur la possible (ou non) alternative entre conflits organisés et non organisés, pour émettre l'idée que le conflit industriel n'est pas un phénomène unitaire mais multidimensionnel, et que l'hypothèse d'un réservoir unique de mécontentement conduisant à toutes les formes d'action, individuelles et collectives, d'absentéisme et de grève, n'est pas correcte. Il examine ensuite, d'un regard toujours critique, l'utilisation statistique du nombre de grèves, sous l'angle sociologique et économique, pour entreprendre après une évaluation de la situation dans les pays membres de l'OCDE à l'aide de tableaux comparés.

D. Weiss compare ensuite différents facteurs, dont l'environnement politique des pays à haut degré de conflictualité et les rapports entre régime politique et taux de grève. Il s'arrête longuement sur le cas de la République fédérale d'Allemagne, pour conclure sur le thème de la banalisation de la grève. Un livre, actuellement sous presse, sur le consommérisme, reprendra dans le détail ce thème, déjà esquissé par $\mathrm{D}$. Weiss dans plusieurs articles et interviews antérieurs, dans lesquels il fut le premier à opérer le rapprochement, pour ce qui est de la situation française, entre ce qu'il appelle système de relations consommériales et le système de relations du travail.

Luigi Mengoni parle des instruments juridiques de règlement du conflit, et traite, à son tour, du cas allemand où, contrairement à la France et à l'Italie, le syndicat a le monopole du déclenchement et de la gestion de la grève, et où l'étroite coordination des modes de grève et de la négociation collective comporte une limite concrétisée dans une symétrie des sacrifices. Les tentatives de réglementer par la loi le droit de grève en Italie ont failli, dit Mengoni. Quant à la jurisprudence, elle a 
largement liberalisé les grèves tournantes et «à hoquet», en même temps qu'elle adoptait un critère moins rigoureux d'évaluation de la licéité du lock-out défensif adopté par l'employeur. Enfin, les formes traditionnelles d'intervention directe pour le règlement des conflits collectifs du travail, la conciliation, la médiation et l'arbitrage sont faiblement institutionnalisées.

Le volume est complété par le texte des débats et par les très intéressantes propositions du groupe des cadres de la Fiat sur les structures de représentation, le referendum préalable au déclenchement de la grève, la rationnalisation des niveaux de négociation et les systèmes de règlement et de «refroidissement» des conflits.

\section{P. JOUBERT}

Institut d'Administration des

Entreprises, Paris

Politica, partidos y sindicatos en la empresa, par Dimitri Weiss, Madrid, Instituto de Estudios laborales y de la Seguridad social, 1982, $136 \mathrm{pp}$. ISBN 84-5007823-7

El poder sindical en Espana, par J.A. Sagardoy Bengoechea et David Leon Blanco, Barcelona, Editorial Planeta/ Instituto de Estudios Economicos, 1982, 210 pp. ISBN 84-320-7318-0.

Voici qu'au cours de l'hiver 1982/1983, à Paris, le Rassemblement pour la République (RPR), présidé par Jacques Chirac, remettait en avant la participation, l'un des vieux thèmes du mouvement gaulliste. Mais la nouveauté issue d'un comité central extraordinaire du mouvement, ouvert à la presse et consacré aux difficultés des salariés dans leur vie professionnelle, tenait à un changement de cap qui, en partant du fait que le parti avait par trop négligé jusqu'alors le travail politique dans les entreprises, plaçait le message politique en direction de celles-ci au premier plan des préoccupations. Aussi, y a-t-on beaucoup parlé des adhérents du RPR dans les entreprises, organisés depuis janvier 1982 dans des «sections RPR d'entreprise», signe de la nouvelle priorité qui leur est accordée.

Presque simultanément, le secrétaire national du Parti socialiste (PS) chargé des entreprises dénonçait, lui, le "double jeu fréquent» des communistes dans les entreprises françaises, estimant, notamment, que «le couple CGT-parti communiste est ambigu» et que la CGT «reprend de plus en plus souvent le ton de la protestation que le PC ne peut assumer aussi ouvertement, du fait de la solidarité majoritaire».

Autre tournant, quelque peu surprenant: la Confédération générale des cadres (CGC), devenue Confédération française de l'encadrement (CFE), jugeait dépassée la notion d'apolitisme, reconnaissant que le syndicalisme est devenu «incontestablement un agent de transformation de la société», mais en ajoutant que «se mettre à la remorque d'un parti politique constituerait une démarche suicidaire».

En Espagne, la victoire aux élections législatives du parti socialiste ouvrier espagnol (PSOE) et la place occupée dans son programme de gouvernement par les relations du travail faisaient rebordir au premier plan les liens, pratiquement organiques, du parti avec "son" syndicat, l'Union générale des travailleurs (UGT), désormais en passe de devenir la première confédération syndicale. Quant à la formation d'extrême droite Fuerza Nueva, qui avait créé son propre syndicat, elle a annoncé sa dissolution et s'est transformée en «association culturelle».

C'est dans ce contexte que paraît à Madrid, en une excellente présentation graphique, dans la collection des monographies de l'Institut d'Études du travail et de la Sécurité sociale (patronné par le ministère du Travail et de la Sécurité sociale), le livre de Dimitri Weiss sur «Politique, partis et syndicats dans l'entreprise».

Écrit dans une perspective de sciences de gestion, que l'auteur définit dès le départ comme étant des «sciences (politiques) de la décision et du gouvernement de l'entreprise», 